\title{
Universidad e investigación científico- tecnológica como motor del desarrollo Propuesta de un Plan de reestructuración de la Universidad de la República (1967)*
}

\author{
University and scientific-technological research \\ as an engine for development \\ Proposal of a Restructuring Plan of the University of \\ the Republic (1967)
}

\begin{abstract}
Maria Laura Martinez
Facultad de Humanidades y Ciencias de la Educación

Universidad de la República mlm@vera.com.uy
\end{abstract}

Recebido em 04/09/2017

Aceito em 17/09/2017
Independencia política, independencia económica, autonomía cultural son tres factores decisivos de la verdadera independencia de las naciones. La independencia política no es mucho más que una ilusión, si no se fundamenta en una verdadera independencia económica. Esta a su vez es sólo posible si existe una autonomía cultural, que a través de la producción de técnicas científicas, posibilite el uso autónomo de los recursos naturales de la nación.

Óscar Maggiolo en Universidad de la República. Facultad de Ingeniería, 2009, p. 69.

\section{Resumen}

Al cumplirse 50 años de la presentación, por parte del Rector Óscar Maggiolo, del Plan de reestructuración de la Universidad de la República, este trabajo se propone revisitar el documento en uno de sus aspectos esenciales: el fomento de la investigación científica como motor del desarrollo nacional. El plan, que exponía los principales desafíos que enfrentaba la institución y planteaba un programa integral de cambio, estaba pensado en torno a la función de producción de conocimientos, como tarea medular de la Universidad, y tenía como eje central el desarrollo de las actividades de investigación pura y aplicada y el fomento de las carreras en ciencias básicas, con el objetivo de promover el desarrollo económico y social del país. Para ello proponía una serie de medidas, como la creación de institutos centrales de investigación, la formación de recursos humanos, el equipamiento instrumental y bibliográfico y la proyección de nuevos edificios universitarios.

Palabras clave: Universidad, investigación científico-tecnológica, desarrollo, Uruguay, plan 


\section{Abstract}

At the 50th anniversary of Rector Óscar Maggiolo's presentation of the University of the Re-public's restructuring plan, this paper intends to revisit the document in one of its essential aspects: the promotion of scientific research as an engine for national development. The plan, which outlines the main challenges faced by the institution and proposes a comprehensive change program, was designed around the role of knowledge production, as a core task of the University, and it was central to the development of pure and applied research activities and to the promotion of careers in basic sciences, with the aim of promoting the country's eco-nomic and social development. To achieve this, it proposes a series of measures, such as the creation of central research institutes, human resources training, instrumental and bibliograph-ical equipment and the design of new university buildings.

Key words: Uruguay, scientific-technological research, development, university, plan

\section{Introducción: Plan de reestructuración de la Universidad (PRU) (1967)}

El Plan de reestructuración de la Universidad (de la República, Uruguay) es un documento que fue preparado con el fin de proponer una base de discusión para la formulación del Plan de Política Universitaria del quinquenio 1968-1972, cuya materialización inmediata fuera la estructuración del Proyecto de Presupuesto de la Universidad de la República (Udelar) que regiría en dicho periodo. El texto se presentó durante el rectorado del ingeniero Óscar Maggiolo (1966-1972), ${ }^{1}$ quien fue su principal propulsor y la figura emblemática de aquel, a tal punto que frecuentemente se le designa como Plan Maggiolo, aunque el documento fue el resultado de un trabajo colectivo.

El plan exponía sintética y claramente los principales desafíos que enfrentaba la institución y planteaba un programa integral de cambio. Fue dispuesto en forma de un conjunto de objetivos, acompañado por una justificación general y una serie de apéndices en los que cada uno de los distintos proyectos describía sus objetivos concretos. Dentro de ellos se mencionaban en detalle aquellos que, por distintas razones, se consideraba necesario promover especialmente, intensificar o directamente comenzar.

Se consideraba que la Universidad debía encarar modificaciones en la propia concepción de su misión y de su estructura. Respecto al primer punto, se buscaba una Universidad más integrada al medio social y más comprometida con el desarrollo de sus funciones básicas: docencia, investigación y extensión. En cuanto al segundo, el principal problema era que la Universidad estaba organizada como una federación de facultades y no como un instituto único. El plan proponía una organización alternativa, con la creación de institutos centrales que tenían una función fundamentalmente investigativa, coordinada con las tareas de docencia y extensión. Creaba además una Facultad de Educación y promovía la integración de todos los niveles de educación del sistema: superior, media y primaria. Dichas modificaciones no podían realizarse sin un adecuado plan de metas. Dos razones exigían una planificación concreta: que los servicios públicos de educación superior se realizaran efectivamente a ese nivel y que el cumplimiento de estas metas demandaría importantes erogaciones que, aunque proporcionales a la capacidad de producción del país, incidirían en su economía en crisis.

La oportunidad de este plan estuvo relacionada con un conjunto de factores internos de la institución, pero sobre todo con la coyuntura nacional y regional. A la interna de la institución había un grupo de universitarios comprometidos con la transformación estructural de la Universidad, especialmente interesados

1 Óscar Maggiolo Campos (1920-1980) fue ingeniero industrial (1947), realizó estudios de especialización en Francia, Inglaterra y EEUU. Fue docente del Instituto de Máquinas y luego director del Departamento de Mecánica de los Fluidos de dicho Instituto de la Facultad de Ingeniería. Integrante del Consejo de la mencionada Facultad (1953, 1959-1963, 1963-1966), presidente de la Comisión de Investigación Científica de la Universidad y rector de la Universidad de la República (1966-1968, 1968-1972). 
en la función social que ella debía cumplir y preocupados primordialmente por la investigación científica y sus repercusiones sociales y económicas. Es decir, el plan no surge de una demanda política externa sino en el seno de una matriz interna favorable al cambio institucional, derivada de cambios instituidos en rectorados anteriores y de los debates emanados de la discusión de la Ley Orgánica de 1958. ${ }^{2}$ En cuanto a la situación regional, el periodo estuvo caracterizado por importantes debates acerca de la importancia de la educación superior para el desarrollo social y económico. Por último, en el ámbito nacional, articulado como un pedido de partidas presupuestales, el plan aprovechaba la coyuntura que ofrecían los nuevos instrumentos de planificación del gobierno bajo el influjo de la Comisión de Inversiones y Desarrollo Económico (CIDE), los lineamientos de la nueva Constitución de la República de 1967 y la disposición del Presidente de la República, Óscar Gestido, ${ }^{3}$ a dialogar con la Universidad. La CIDE era un organismo técnico, surgido en 1960 con el objetivo de "formular planes orgánicos de desarrollo económico, proyectar y buscar su financiación interna y externa, coordinar toda la labor tendiente a aumentar la productividad nacional y vigilar la puesta en práctica de los planes que se apruebe"4 (Garcé, 2002, p. 48). Bajo su influjo se creó la Oficina de Planeamiento y Presupuesto (OpP) del Poder Ejecutivo, de la que provino la directiva de elaborar presupuestos por programas, es decir, de asociar las solicitudes de partidas presupuestales a cronogramas de actividades para alcanzar determinados objetivos. El PRU se adaptó a ese modelo, a pesar de que Maggiolo estaba fuertemente en desacuerdo con las conclusiones del Informe sobre el estado de la educación en el Uruguay. Plan de desarrollo educativo (CIDE, 1961), respecto a la educación superior (se oponía a que el presupuesto básico de la Universidad debiera adaptarse a un desarrollo definido en consonancia con directivas norteamericanas y era contrario a limitar el ingreso de estudiantes, entre otros puntos). A pesar de estos desacuerdos, Maggiolo compartía algunas de las sugerencias de la CIDE como las correspondientes a las políticas de becas, la alta dedicación docente, la formación terciaria en educación, la integración vertical del sistema educativo.

El PRU sufrió resistencias y críticas dentro de la propia institución, tanto de sectores conservadores como progresistas, largas discusiones que mostraron la dificultad para pasar del nivel del pedido presupuestal al de una verdadera discusión sobre la necesidad de transformación universitaria. La crítica situación económica de la institución y la premura por cumplir con los plazos de remisión de la solicitud presupuestal al Gobierno, determinaron que el planteo del plan no pasara de obtener un apoyo en términos generales, pero no fuera discutido a fondo y en detalle. Por otra parte, el Poder Ejecutivo le otorgó a la Universidad sólo la mitad del presupuesto solicitado, dejando en claro con ese gesto que no apoyaba la reforma en la educación superior del país. Por último, a fines de 1967, muere el presidente de la República Óscar Gestido y la Universidad pierde toda esperanza de lograr un ámbito de apertura y colaboración con el gobierno. Luego de la asunción del vicepresidente Jorge Pacheco Areco, ${ }^{5}$ si bien algunos sectores siguieron discutiendo algunos aspectos del plan, rápidamente la Universidad debió preocuparse por sobrevivir a las violaciones de su autonomía, las interrupciones de cursos, las clausuras de locales, las amenazas de intervención, los enfrentamientos con las fuerzas represivas estatales y hasta la muerte de estudiantes. En ese contexto, la reforma estructural dejó de estar en el primer lugar de la agenda universitaria y se convirtió en un conjunto de aspiraciones truncadas por el golpe de Estado de 1973.

A pesar de la inviabilidad del plan, en tanto que sigue siendo fuente de inspiración para los universitarios uruguayos, como uno de los proyectos más ambiciosos de reforma integral de la Universidad de la República desde su fundación, al cumplirse cincuenta años de su presentación consideramos oportuno revisitarlo en

2 Ley Orgánica de la Universidad de la República, n. ${ }^{\circ}$ 12549, del 29 de octubre de 1958.

3 Óscar Gestido (1901-1967), era militar y político, presidente de la República Oriental del Uruguay desde el 1..$^{\circ}$ de marzo al 6 de diciembre de 1967.

4 La CIDE fue creada el 27 de enero de 1960 por un decreto dictado por el ministro de Hacienda, presidida por el propio ministro e integrada además por los ministros de Obras Públicas, Industrias y Trabajo, Ganadería y Agricultura, por el contador general de la Nación, el director de Crédito Público y un director del Banco de la República. Un nuevo decreto del 17 de marzo de 1960 modificó esta integración inicial, incorporando al ministro de Relaciones Exteriores y al presidente del Concejo Departamental de Montevideo.

5 Jorge Pacheco Areco (1920-1998), era político y periodista, presidente de la República Oriental del Uruguay desde el 6 de diciembre de $1967 \mathrm{al} 1 .^{\circ}$ de marzo de 1972. Su gobierno estuvo signado por la aplicación de medidas prontas de seguridad para reprimir las protestas sociales. 
uno de sus aspectos esenciales: el fomento de la investigación científica en la Universidad como motor del desarrollo nacional.

\section{Del modelo profesionalista a la profesionalización científica}

La Universidad de fines de 1950 y de la década de 1960 se caracterizó por albergar en su seno a docentes e investigadores que defendían el rol de las instituciones de educación superior como promotoras del desarrollo económico y social de sus naciones y a la investigación científico-tecnológica como la llave para el logro de dicha misión. Además de la defensa de los principios de autonomía y cogobierno, consagrados en la Ley Orgánica de 1958, esta generación de intelectuales reformistas apostaba por la renovación de la educación superior y la instauración de la institucionalidad científica como elementos primordiales para el cambio social.

El ingeniero Maggiolo, integrante de ese núcleo de intelectuales reformistas, defendía - en ocasión de comentar la Ley Orgánica de la Universidad en el editorial de la Revista de la Asociación para el Progreso de la Ciencia (1958) - la necesidad fundamental de incrementar la actividad científica. Si bien la Universidad cumplía decorosamente con la función docente de preparar profesionales, no sucedía lo mismo con respecto a su misión de ser un centro activo en materia de pensamiento original, es decir, de creación de conocimientos. Maggiolo entendía que el factor primordial en que se asentaba el prestigio de una Universidad era el de los trabajos originales que los miembros de su personal docente publicaban regularmente (1958, p. 1-2).

Nuestra Universidad, según Maggiolo, no alcanzaba el mínimo exigible en el campo de la investigación científica, aun reconociendo que la poca que se realizaba en el país se llevaba a cabo casi en su totalidad dentro de dicha institución. Por ese entonces, en esta última se transitaba un proceso de crítica y descomposición del ideal profesionalista, formador de los cuadros profesionales de dirección, aspecto que la había caracterizado hasta entonces. ${ }^{6}$ Ideal que junto con el retraso científico-tecnológico de la región y otros factores culturales y políticos motivaron la postergación de la incorporación de la función investigación en la universidad latinoamericana (Latapí, 1976, p. 24).

Los sectores reformistas de nuestra universidad defendían, por su parte, la necesidad de concentrar esfuerzos en los estudios básicos que permitieran una constante adaptación del experto al avance del conocimiento y en la profesionalización de los investigadores. La creación de nuevos planes de estudio estaba presidida por la oposición a la concepción profesionalista que defendía la enseñanza práctica de resultados inmediatos.

Este proceso de reforma, comenzado posteriormente a la segunda Guerra Mundial, adquirió un énfasis importante a partir del rectorado de Mario Cassinoni ${ }^{7}$ (1956-1964) quien, junto con su equipo, se propuso como objetivos principales: superar la mera formación de profesionales ampliando las funciones universitarias, priorizar la investigación científica y establecer el régimen de gobierno coparticipativo para la institución. Se pretendía procesar los cambios que permitieran a la Universidad contribuir al desarrollo y progreso de la sociedad en sus múltiples aspectos y cumplir su misión social impulsando el desarrollo de las investigaciones y las carreras más vinculadas a la problemática económica del país. Con Cassinoni en la dirección de la Universidad se adoptó una política clara en ese sentido y la insistencia en la necesidad del régimen de dedicación total y su ampliación fueron pasos decisivos, aunque no suficientes. ${ }^{8}$

La investigación científica y el binomio docencia-investigación se constituyeron para las autoridades de la Universidad en preocupaciones relevantes y en eje del programa del desarrollo universitario desde el

6 La Universidad había encarado en 1945 un nuevo tipo de enseñanza, que superara el profesionalismo estrecho, con la creación de la Facultad de Humanidades y Ciencias, pero lo había hecho sobre la base de opciones que le venían de fuera, con las consiguientes imposiciones, y a base de ideas caducas, socialmente inviables(Otero, 1969, p. 21).

7 Mario Cassinoni (1907-1965), era doctor en medicina (1937), fue decano de la Facultad de Medicina (1949-1950; 1950-1953), rector de la Universidad de la República (1956-1964), doctor Honoris Causa de la Universidad de la República (1965) y diputado por el Partido Socialista (1954-1956).

8 Según el informe de la Comisión de Investigación Científica de la Universidad de la República al rector Cassinoni en 1957, a diez años aproximadamente de la creación del régimen de dedicación total, había en la Facultad de Medicina 17 docentes investigadores que trabajaban en ese régimen, en la de Ingeniería sólo 5 y ninguno en las demás (Cassinoni, 1962, p. 239). 
comienzo de la reforma. En el informe de la Comisión Central de Investigación, creada en 1957 especialmente para abordar el tema de la investigación en dicha institución, se sostenía que Uruguay debía hacer los esfuerzos necesarios para estar al día e impulsar nuevas ramas de estudios, para lo cual se debía integrar las cátedras en base a institutos y desde ellos proyectar una política de investigación que favoreciera la economía del país.

El fomento de la investigación se fue acelerando poco a poco en la Universidad y hubo algunos aportes especialmente valiosos. Por ejemplo la labor del Seminario de Estructuras Universitarias, dirigido por Darcy Ribeiro ${ }^{9}$ (1967), que convocó a un importante número de dirigentes universitarios y miembros de la federación estudiantil, en vistas de la inmediata reforma universitaria.

A nivel nacional, mientras tanto, luego de desarticulada la política científico-tecnológica del segundo periodo batllista (1911-1915) — que buscaba la independencia científica, tecnológica y energética del país a partir de instituciones como las creadas por el denominado Proyecto Eduardo Acevedo - ${ }^{10}$ los objetivos se modificaron en procura de la superación del atraso económico y en un intento por alcanzar determinados niveles de desarrollo. A partir de una reestructura del sistema científico, que tenía como fin asimilarse fundamentalmente al modelo norteamericano, se impuso la necesidad de que los científicos transitaran un proceso curricular planificado bajo ciertos modelos normalizados fuera del país, reproduciendo las condiciones y la estructura del sistema científico internacional, promoviéndose así el desarrollo de la profesionalización científica.

Para lograr estos cambios eran necesarias acciones provenientes tanto desde el Gobierno Nacional como desde el Consejo Directivo Central de la Universidad. Este último debía comenzar por poner en práctica normas elementales de estímulo a la investigación científica, tales como ampliar el número de docentes con dedicación total, extender la jornada de trabajo en los laboratorios, facilitar instrumental y colaboradores a los investigadores que presentaran planes serios de investigación científica, detener el éxodo de los pocos investigadores que se tenían, crear becas internas y externas para jóvenes con entusiasmo por la ciencia, amparar a su vuelta al país al investigador que se había doctorado en centros del exterior, contratar científicos extranjeros en aquellas disciplinas básicas que no se cultivaban adecuadamente en el país, consagrar el reconocimiento decisivo de los méritos científicos para el ascenso en la carrera universitaria, simplificar los trámites para la adquisición de equipos e instrumentos, reducir la burocracia contable, y por último, pero posiblemente una de las medidas más importantes, crear las carreras científicas básicas.

En ese momento, la industria nacional, tanto oficial como privada, recurría poco al método científico para resolver sus problemas de producción y desarrollo. Prefería comprar patentes viejas o recurrir a los subsidios y barreras aduaneras, lo que representaba sumas de dinero perdidas para la economía nacional, pero que destinadas al subsidio de laboratorios traerían aparejado un mejoramiento de la crítica situación económica y social y un estímulo a las carreras científicas.

Maggiolo denunciaba una política planificada para propiciar el desarrollo del país supeditado a las inversiones de las empresas norteamericanas, que pretendía darle a la enseñanza superior el papel de producir

9 Darcy Ribeiro (1922-1997) fue antropólogo y cientista social, primer rector de la Universidad de Brasilia, expulsado por la dictadura brasileña se exilió en Uruguay entre los años 1964 y 1968. Se relacionó con muchos intelectuales uruguayos, ejerció la docencia en la Facultad de Humanidades y se vinculó a la Universidad de la República en su conjunto, donde dictó en 1967 un "Seminario de estructuras universitarias" sobre las modificaciones que se debían realizar a un modelo de universidad latinoamericana basado en el patrón europeo y que se adaptaba cada vez menos a las necesidades del continente. En consonancia con las directivas que se despliegan en el PRU de 1967, Ribeiro vislumbraba la transformación de las universidades latinoamericanas a partir de su compromiso con la producción científica, que les permitiera convertirse en generadoras de cambio social y promotoras de autonomía de sus países.

10 A principios del siglo xx, durante la segunda presidencia de José Batlle y Ordóñez (1911-1915) y con el Ministerio de Industrias a cargo de Eduardo Acevedo (1911-1913), el Gobierno uruguayo se propuso dar al país una capacidad científico-tecnológica autónoma, mediante la creación de instituciones técnico-científicas y estimulando la formación de comunidades científicas nacionales. En ese contexto fueron creadas cuatro instituciones entre 1911 y 1912 que fueron primordiales en dicha estrategia: 1) Las Estaciones Agronómicas, fundadas en 1911 con el fin de educar a los hijos de los estancieros y agricultores en las modernas técnicas de explotación rural, y servir de modelo a productores desarrollando cultivos inteligentes y seleccionando las razas ganaderas más aptas. 2) El Instituto de Pesca, creado el mismo año, destinado a explotar una riqueza aún inexplorada. 3) El Instituto de Geología y Perforaciones, fundado en 1912 con el objetivo de hacer la prospección de petróleo. 4) El Instituto de Química Industrial, creado junto al anterior con el fin de investigar sobre el combustible nacional y la utilización de materias primas del país. 
sólo los profesionales necesarios para el mantenimiento de las industrias y empresas, de acuerdo con las directivas venidas desde el exterior, o a lo sumo el papel de desarrollar investigación aplicada que podría ser beneficiosa para dichas empresas extranjeras. Esta política se volvía evidente cuando el país pretendía que la Universidad se transformara, que pasara de ser un instituto puramente formador de profesionales a ser un instituto de investigación y formación de investigadores, cuando se planteaba el problema de la investigación en las ciencias básicas, que era precisamente el campo que una vez desarrollado le daría al país una posibilidad de independizarse efectivamente (Universidad de la República. Archivo General, 2010, p. 92).

\section{3. Óscar Maggiolo, propulsor de la investigación científica y promotor de la reforma}

Óscar Maggiolo fue un tenaz propulsor del desarrollo de la ciencia y la tecnología, dentro y fuera de la Universidad.

Además de docente e investigador en régimen de dedicación total de la Facultad de Ingeniería, miembro del Consejo de dicha facultad en varios períodos, presidente de la Comisión Central de Investigación Científica de la Universidad y finalmente, rector de dicha casa de estudios, Maggiolo tuvo un rol fundamental en instituciones nacionales dedicadas al estímulo y promoción de la ciencia y la tecnología, en las que defendió varias de las ideas que luego se vieron plasmadas en el $P R U$.

Es el caso de su participación en la propia Facultad de Ingeniería, la Asociación Uruguaya para el Progreso de la Ciencia (AUPC) y el Consejo Nacional de Investigaciones Científicas y Técnicas (CONICYT), entre otras.

Desde la Facultad de Ingeniería, en el año 1944 y en medio de una polémica por la creación de los institutos de ciencias básicas en dicha facultad, Maggiolo opinaba, junto con otros docentes, que no todo se arreglaba con un cambio de plan de estudios, sino que se debía lograr también, simultáneamente, un cambio en el espíritu de la enseñanza. (Maggiolo, Posada, Martínez, y Bouton, 1944). Esta Facultad, en particular, debía dejar de pensar en sus egresados meramente como profesionales liberales, que trabajaban solamente para los grandes proyectos del Estado. Para ello era necesario, en primer lugar, incrementar y fomentar la investigación científica entre el personal docente de la Facultad. En segundo lugar, era necesario que los profesores de las materias básicas, salvo aquellas que por su índole particular requirieran experiencia profesional, tuvieran dedicación exclusiva. Del mismo modo, era necesario conceder permisos periódicos al personal científico y docente para que hicieran, en el país o en el extranjero, trabajos de investigación o perfeccionamiento en sus respectivas especialidades. Estas ideas, como veremos, se vieron reflejadas luego como un desiderátum para la Universidad toda en el plan de 1967.

En esa misma década, más precisamente en 1942, fue creada la Junta Coordinadora de las Investigaciones de los Institutos y Laboratorios de la Facultad de Ingeniería, cuyos cometidos eran relacionar y facilitar las investigaciones, estudios y trabajos científicos relacionados con las funciones de institutos y laboratorios, constituir el enlace entre los investigadores y los profesores de la Facultad, estudiar todas las cuestiones de carácter general que contribuyeran al progreso científico e industrial del país, asesorar al gobierno nacional $\mathrm{y}$ formar anualmente una lista de trabajos, entre otros.

En ese contexto, Maggiolo defendió que el asesoramiento industrial podía resolver el problema de la financiación de los institutos, dado que para conseguir los equipos de laboratorio y programar experiencias en el campo de la ingeniería era necesario disponer de enormes sumas de dinero, que no resultaban fáciles de obtener del Estado. A favor de esto se debía comenzar un movimiento ante los industriales y ante los entes industriales del Estado. Había que mostrarle al industrial lo que la Facultad con sus institutos podía hacer por él y por su negocio. Nuestra industria era una industria prestada, que dependía de los resultados de las investigaciones que se llevaban a cabo en el extranjero, no tenía independencia (Universidad de la República. Facultad de Ingeniería, 2009, p. 13-16).

Por otra parte, desde la Cámara de Industrias de Uruguay también se planteó la necesidad de crear un laboratorio de investigación tecnológica que estuviera al servicio de las industrias del país. Dicha institución 
había recibido el ofrecimiento de algunas reparticiones técnicas que contaban con laboratorios de ese tipo. Entre ellas, estaban la Facultad de Ingeniería y la Fundación Armour ${ }^{11}$ para poner en práctica el Plan Armour de Ayuda Técnica Internacional, tanto como servicio al programa nacional como a las industrias privadas.

En 1952 la Cámara de Industrias junto con la Facultad inició las conversaciones sobre el establecimiento de ayuda técnica con base en los institutos de aquella casa de estudios, por lo que se resolvió hacer las gestiones ante la Armour a través de la Comisión Interministerial de Asistencia Técnica; ${ }^{12}$ esto resultó en la concertación de un convenio con la mencionada Fundación, a fin de obtener de ella un asesoramiento previo sobre la organización del proyectado servicio de investigaciones y la preparación de un programa de fomento de la productividad de la industria nacional, especialmente de la pequeña y mediana empresa.

La Fundación Armour, en el informe final de su proyecto n. ${ }^{\circ}$ I 514-4, denominado Avaluación de laboratorios técnicos y estudio tecnológico preliminar del Uruguay, concluyó que la aplastante evidencia obtenida marcaba la necesidad de crear un centro independiente dedicado exclusivamente a la investigación técnica y al desarrollo para la industria y el gobierno. Para ello los miembros de la misión planearon una organización y métodos de funcionamiento para el futuro centro, según lo cual la vinculación entre este y los institutos de la Facultad de Ingeniería sería solamente de carácter funcional y aplicable si se concertaba un acuerdo de colaboración entre ambas partes.

El Centro de Asistencia Técnica debía ser, de acuerdo a este informe, independiente de todo organismo oficial y dedicarse exclusivamente a la investigación técnica; era unánime la opinión de los expertos en cuanto a separar de la órbita de la Universidad a la institución que se deseaba crear. De todas maneras, se preveía la cooperación efectiva de los institutos de la Facultad de Ingeniería, ya que tenían la mayor variedad de equipos para ensayos tecnológicos, por lo que se proponía instalar el centro en las proximidades de esa institución o en su mismo edificio, de ser posible. Esta conclusión venía a modificar sustancialmente los puntos de vista iniciales, que incluían la intervención inmediata de los institutos y desplazaba a la Universidad del proceso de producción y aplicación industrial de la tecnología, así como bloqueaba su participación institucional en la administración del centro proyectado. Lo que había sido concebido como un mecanismo para tratar de darle más recursos a la Universidad a través del asesoramiento a la industria, se había vuelto una forma de quitarle fondos.

En 1956 se publicitó el informe de la Armour y los consejeros de la Facultad de Ingeniería, Julio Ricaldoni, ${ }^{13}$ Vicente I. García ${ }^{14}$ y Óscar Maggiolo elaboraron un memorándum titulado Consideraciones sobre la creación del Centro de Investigación Tecnológica, que impugnaba sus conclusiones y sostenía la necesidad de que el Instituto de Investigación Tecnológica fuera de carácter nacional y universitario, agrupando a todos los laboratorios de las facultades que pudieran colaborar.

11 La Armour Research Foundation del Instituto de Tecnología de Illinois es "una organización no lucrativa para proveer investigaciones confidenciales e independientes, desarrollos e ingeniería experimental al servicio de la industria, el gobierno y el público en general [...]. La Fundación reconoce que la investigación es un camino que conduce al desarrollo y a la prosperidad [...]. Los 6 Departamentos de Investigación de la Fundación son: Física, Metales, Química e Ingeniería, Química Aplicada, Ingeniería eléctrica, Cerámicas y Minerales. [...]. La División Internacional de la Fundación trata de proveer a otros países del mismo servicio de investigación que se efectúa en Estados Unidos, el cual no es lucrativo y es apolítico. El valor de esta investigación internacional ha sido demostrado en la creación de nuevos fondos de divisas-dólar, descubrimiento de fuentes naturales no desarrolladas, selección de industrias solventes, alentamiento de inversiones, asesoramiento de personal técnico en varios países y establecimiento de facilidades para la aplicación de las investigaciones" (Memoria, Armour Research Foundation, Plan Armour de Ayuda Técnica Internacional al Uruguay, 1950, p. 673).

12 En ella estaban representados todos los ministerios con injerencia en los problemas de que se tratara y podían enviar representantes también los entes autónomos interesados en la materia. Era la encargada de coordinar los planes y estudios relacionados con la asistencia técnica elevando sus informes al Poder Ejecutivo por intermedio de la secretaría de Estado correspondiente.

13 Julio Ricaldoni (1906-1993), era ingeniero civil (1933), fue director del Instituto de Estática (1941) y del Instituto de Estructura y Transporte (1986-1993), profesor de Estructuras Metálicas y de Madera (1935-1953), de Puentes y Grandes Estructuras (1953), de Resistencia de Materiales (1951-1958) en la Facultad de Ingeniería. Docente de Mecánica Aplicada a la Construcción en la Facultad de Arquitectura (1949-1952), decano de la Facultad de Ingeniería (1969-1973, 1985) y doctor honoris causa de la Universidad de la República (1986).

14 Vicente I. García, era ingeniero civil (1905), fue docente, director del Instituto de Materiales de la Facultad de Ingeniería, decano de la Facultad de Ingeniería en varios periodos (1928-1931, 1931-1934, 1939-1940, 1940-1943). 
Que Uruguay necesitaba un centro de investigaciones de ese tipo no era tema de discusión, "pero ninguna institución seria [decía el informe] puede sostener que es abrumadora la evidencia de que la investigación industrial no puede realizarse en los institutos universitarios" (García, Maggiolo y Ricaldoni, 1956, p. 6).

El informe sostenía que no era conveniente crear un instituto tecnológico independiente porque: a) Uruguay no podía, en lo financiero, sostener dos institutos de investigación tecnológica, uno por la industria y otro por el Estado; b) sería imposible conseguir en el país personal científico con experiencia en investigación fuera de la Universidad. Una de las dos instituciones se quedaría sin recursos humanos; c) la necesidad de que el laboratorio de investigación funcionara bajo un régimen de dedicación total de su personal era indiscutible y con más razón lo era para los institutos universitarios; d) un instituto tecnológico independiente de la Universidad y con laboratorios propios mataría incuestionablemente los de esta institución porque todos los recursos por proventos e impuestos serían destinados fundamentalmente a los laboratorios de aquel centro; e) ningún instituto o laboratorio universitario podía tener como única meta los ensayos rutinarios tal como proponía Armour; f) la creación del centro en el seno de la Universidad beneficiaría su autonomía, en cuanto ya no dependería exclusivamente del presupuesto estatal (Ibid, p. 7-13).

A pesar de esto, el 9 de noviembre de 1956 el Consejo de Gobierno envió un mensaje a la Asamblea General proponiendo la creación del Centro de Asistencia Técnica para la Industria bajo la órbita del Ministerio de Industrias y Trabajo, ${ }^{15}$ proyecto que fue impugnado por la Facultad de Ingeniería mediante una declaración pública. ${ }^{16}$

$\mathrm{Al}$ año siguiente, y en vistas de que había tomado fuerza la idea de algunos industriales de crear un centro pagado y gobernado por ellos mismos, la Facultad presentó un anteproyecto de estatuto para el Centro de Asistencia Técnica, redactado por los consejeros García, Ricaldoni y Maggiolo, en el que se pronunciaba a favor de crearlo dentro de la órbita de la Universidad. Según el anteproyecto, el Centro de Asistencia Técnica para la Industria (CATI) era un organismo creado por la Universidad con el fin de colaborar, por medio de la investigación industrial, con el progreso y el desarrollo de la industria nacional, desenvolviendo su actuación con autonomía técnica y funcional. ${ }^{17}$

La Universidad encontraba serias dificultades para hacer la investigación científica a la que estaba llamada. La formación de un centro de asistencia para la industria en la Facultad habría ayudado a resolver ese problema. Pero no sólo eso, le habría permitido mantener una relación mucho más sólida con el aparato productivo del país. Un aparato productivo que, por otra parte, no estaba exento de problemas. Las industrias uruguayas de aquel momento eran

[...] más que fábricas y talleres, verdaderos museos de tecnología, con maquinaria obsoleta en la mayoría de las factorías textiles, en las productoras de alimentos y bebidas, en los talleres metalmecánicos o en la industria naval (Universidad de la República. Facultad de Ingeniería, 2009, p. 249).

La única solución es desarrollar una tecnología propia, independiente, adecuadamente basada en el estudio científico de métodos de fabricar productos por medio de una industria autóctona. No es una solución importar 'ciencia y tecnología' como quien importa automóviles o vagones de ferrocarril (Ibid, p. 35).

Para lograr esa industria autóctona era imprescindible un centro de asesoramiento industrial con los recursos adecuados y la autonomía necesaria del exterior.

15 Se destinarían 300 millones de pesos anuales para el funcionamiento de dicho centro, cuyo establecimiento y organización quedaba a cargo de la Comisión Especial creada por decreto del 3 de octubre de 1952.

16 Universidad de la República. Facultad de Ingeniería y Ramas Anexas (Uruguay) (1956), Actas del Consejo Directivo, sesión del 13-11-1956, folio 6177.

17 El cati finalmente no se formalizó. Sin embargo, esta polémica puede ser señalada como un antecedente de la creación del Laboratorio de Análisis y Ensayos (1965), luego Laboratorio Tecnológico del Uruguay (LATU), instituciones de asesoramiento industrial independientes de la Universidad. 
Pero no solamente la Universidad, y en particular la Facultad de Ingeniería, hacían énfasis en la necesidad de una política de estímulo al desarrollo científico-tecnológico nacional. Este planteo se hacía también simultáneamente en otras instituciones, como la AUPC.

La AUPC fue creada en Montevideo en diciembre de 1948, entre otros importantes profesionales e investigadores por el ingeniero Maggiolo, que ocupó el cargo de secretario durante varios años. De acuerdo a sus estatutos la Asociación tendía fundamentalmente a "favorecer el progreso de la ciencia" estimulando la investigación científica, facilitando el desarrollo de todas sus ramas.

En esa línea, en la década de 1950, desde la Asociación y en conjunto con la Universidad de la República, se hacía un fuerte énfasis en la necesidad del régimen de dedicación total para los investigadores. La investigación debía ser una profesión, el investigador debía estar dedicado a ello, no ser un aficionado.

En ese marco, desde las páginas del Boletín de la Asociación Uruguaya para el Progreso de la Ciencia primero, y la Revista de la Asociación Uruguaya para el Progreso de la Ciencia después, Maggiolo defendía nuevamente la necesidad de invertir en ciencia y tecnología.

El primer número de esta publicación, que era la vanguardia de la ideología científica en el país y en la Universidad de ese momento, se inicia con un artículo de Maggiolo titulado "La ciencia, la técnica y la sociedad actual", donde plantea por qué un país debe tener un sistema científico. Según Maggiolo, se debía invertir tanto en ciencia pura como aplicada, y en este sentido expresaba lo siguiente:

Es necesario comprender que la inversión que se realice en este campo es dinero bien colocado del cual depende nuestro futuro como sociedad libre y culta de ser respetada en el campo internacional. El pueblo uruguayo y especialmente sus gobernantes deben poner plena atención a este hecho, pues en nuestro país es mucho lo que falta hacer en este sentido (Maggiolo, 1952, p. 11).

Para ello había que comenzar por poner atención en niveles anteriores de educación. En el editorial de 1953, "La enseñanza de la ciencia en el ciclo secundario", Maggiolo comentaba:

Los cometidos fundamentales de la enseñanza media en una democracia deben ser, aparte de la educación moral, el asegurar el desarrollo intelectual de manera que los estudiantes aprendan a estudiar por sí solos, a observar con precisión, a razonar de manera coherente y reflexiva, a valorar objetiva y críticamente las opiniones de los demás y a formarse un juicio propio sobre los problemas fundamentales de la sociedad y los que le interesen personalmente; $\mathrm{y}$ adquirir un cuadro vivo y coherente del mundo en que tendrán que vivir (Maggiolo, 1953, p. 1).

Esa era la finalidad de la enseñanza media, y los recursos materiales necesarios para llevar a cabo esta tarea eran: laboratorios, pequeños talleres y buenas bibliotecas, filmes científicos y diapositivas para ilustrar y complementar explicaciones, además de fomentar en los liceos la formación de clubes de ciencia en los que los alumnos pudieran perfeccionar sus conocimientos y cultivar su curiosidad e imaginación efectuando observaciones y experiencias planeadas por ellos mismos. Y en el número siguiente, Maggiolo expresaba, también en el editorial "Recursos y hombres para la investigación científica":

La investigación científica, sea pura o aplicada, necesita tres condiciones para poderse realizar en forma eficiente: primero, personal científico auxiliar en número necesario, bien preparado y bien seleccionado para la tarea a que se le destine; segundo, recursos financieros amplios para las adquisiciones del instrumental, hoy día costoso en general, que demanda la investigación, tercero, locales apropiados para asiento de la tarea de investigación (Maggiolo, 1955, p. 1). 
Llegaba a la conclusión de que en nuestro país casi todo el dinero destinado a la investigación se invertía en edificios, pero

[...] es imposible equipar esos edificios en instrumental y personal. Hay poco número de plazas y con pocas horas de dedicación. También es malo el método con que se selecciona el personal (concurso de oposición) porque un investigador no se improvisa de la noche a la mañana (Ibid).

Por tanto, era de dudosa utilidad dedicar sumas cuantiosas para edificios universitarios y laboratorios, si no se proporcionaban simultáneamente recursos para personal y equipos que trabajaran dentro de ellos jornadas completas. Aun así, no bastaba solucionar esto, sino que había que cambiar el sistema de selección de investigadores, de modo que los méritos que realmente se tuvieran en cuenta fueran los trabajos científicos originales y la formación.

En esa década, ya nadie dudaba de la necesidad de una ciencia profesionalizada para el desarrollo del país, pero paradojalmente nadie estaba dispuesto a pagar por ella, por tanto, la única solución posible era el apoyo estatal o internacional. A mediados de ese decenio la AUPC en un último impulso antes de disolverse - en 1960-, y siguiendo la experiencia internacional y latinoamericana, propuso la creación de un centro estatal de promoción de la investigación y desarrollo científico, un CONICYT. ${ }^{18}$ Se lo proponía por dos razones fundamentales: todo país avanzado debía tener un consejo que regulara o coordinara la investigación en ciencia y tecnología y había que conseguir financiación para dicha investigación.

En 1961, el Poder Ejecutivo incluyó en la propuesta de Ley de Rendición de Cuentas la creación del mencionado centro (СОNICYT), dirigido por un directorio honorario integrado por cuatro delegados universitarios y siete delegados de ministerios del Poder Ejecutivo. ${ }^{19}$ La Universidad objetó esta forma de creación porque suprimía la posibilidad de discutir acerca de su integración y funcionamiento y porque la excluía del debate. Finalmente, en 1964, fueron designados los cuatro delegados que representaban otras tantas áreas científicas (ciencias agrarias, ciencias sociales, tecnología y medicina) y entre los cuales se contaba el ingeniero Maggiolo.

El hecho de enviar delegados al CONICYT determinó que la Universidad se planteara explícitamente, por primera vez, cuál era la política de investigación científica de la institución y que definiera su política sobre los puntos que se detallaban en el informe solicitado. El informe fue redactado por la Comisión de Investigaciones Científicas de la Udelar y mostraba el estado del problema de la investigación científica en la institución. En este se señalaba su apoyo a los proyectos de interés nacional que se presentaran en los ámbitos de investigación del Estado, universitarios o extrauniversitarios, que a la vez persiguieran objetivos de ciencia aplicada al desarrollo o mejoramiento de la producción agropecuaria, la producción extractiva, la producción industrial, la salud pública y la administración pública. Tal preferencia no excluía el apoyo a proyectos que se presentaren por particulares o que persiguieran objetivos de ciencia pura, fueran naturales o humanistas. No había actividad científica que quedara excluida del interés de la Universidad, fuera de ciencia básica o aplicada; ningún instituto ni área de conocimiento.

El informe mencionaba además que, tratándose de sentar las bases de una actividad orgánica en el campo de la investigación científica, era imperativo en los primeros tiempos dedicar esfuerzos y recursos

18 Los Consejos Nacionales de Investigación son organismos destinados fundamentalmente a asesorar al Gobierno en su política científica, además de tener un papel ejecutivo de apoyo, financiación y desarrollo de la ciencia y la tecnología. Las primeras instituciones de este tipo se crearon luego de la primera guerra mundial en Reino Unido, Estados Unidos, Alemania y URss. En Latinoamérica las primeras en crearse son la argentina y la brasileña, en 1951.

19 Ley 13032 del 7 de diciembre de 1961, artículo 240. Según dicho artículo el CONICYT tendría por cometido promover y estimular el desarrollo de las investigaciones en todos los órdenes del conocimiento; administrar y distribuir los fondos que se le destinaban, pudiendo hacer las adjudicaciones que entendiere convenientes en favor de particulares, funcionarios públicos o instituciones nacionales, públicas o privadas, de cualquier naturaleza. El Consejo fue creado en concordancia con las resoluciones adoptadas por la Conferencia General de la Unesco en el campo de la política científica y las recomendaciones establecidas en las reuniones regionales de dicho organismo: Conferencia de expertos científicos de América Latina (1948) y Seminario sobre la organización de la investigación científica en América Latina (1960). 
a la capacitación general en el país para esta misión. El Consejo Nacional debería encarar de inmediato un relevamiento de la actividad científica nacional existente, debería fomentar la cultura científica, tanto en el plano de la enseñanza pública, desde la escuela hasta la Universidad, como en el ámbito de la administración popular o el de los gobernantes y dirigentes de la administración pública y la economía nacional. Por tanto, había que presentar una vasta toma de conciencia de la investigación científica como factor de desarrollo; propiciar la elaboración de investigaciones tanto en centros nacionales, fundamentalmente universitarios, como en el exterior; realizar una organización sistemática, en gran escala, de la documentación científica, del equipamiento instrumental de los centros básicos nacionales de investigación, universitarios y extrauniversitarios, y otorgar amplio apoyo financiero para la publicación científica.

Como representante de la Udelar en el conicyt, Maggiolo defendió la idea de la autonomía de este organismo con relación al poder político. Si bien las definiciones sobre políticas científicas y la búsqueda de soluciones a la crisis estaban íntimamente relacionadas, no por ello la toma de decisiones al respecto debía estar supeditada a las inclinaciones del gobierno de turno. La autonomía no se dio en ese sentido y el Consejo fue creado en relación de dependencia con el Ministerio de Instrucción Pública y Previsión Social. En 1966, Maggiolo y los otros dos delegados de la Universidad (Carlos Quijano ${ }^{20}$ y Hermógenes Álvarez ${ }^{21}$ ) renunciaron por las injerencias del ministro Juan Pivel Devoto en las decisiones del Consejo y reiteraron su decepción sobre el funcionamiento de este bajo la órbita del Ministerio mencionado, manifestando nuevamente que cuando actuaba como órgano ejecutivo, el CONICYT debía gozar de total autonomía. Lo contrario era propiciar una situación que conspiraba contra el posible éxito de la gestión del organismo.

La participación de Maggiolo en estos ámbitos, su trayectoria de preocupación y defensa de la educación superior y el avance de la ciencia y la tecnología en el país, lo sindicaron, en su momento, como el universitario más indicado para impulsar ese avance y revitalizar las fuentes productivas del país. Esa fue su pretensión al proponer el PRU en 1967.

\section{La investigación científica como eje central de la reforma}

Frente al panorama descripto en los apartados anteriores, cuando Maggiolo accedió al rectorado en 1966 se propuso que la Universidad realizara un esfuerzo importante en el desarrollo de las actividades de investigación científica pura y aplicada, así como en el fomento de las carreras en ciencias básicas durante el quinquenio 1968-1972, esperando que al finalizar este período se hubiese creado la estructura fundamental para encarar con autonomía el desarrollo futuro. Una de las bases fundamentales de la independencia económica de las naciones residía en disponer de una capacidad autónoma para desarrollar las técnicas más adecuadas para la explotación racional de los recursos naturales y la elaboración de productos comercializables en los mercados internos y externos.

Todo ello, sin desmedro de que la investigación científica, como actividad creativa, era inseparable de la función universitaria.

Era indiscutible, sostenía Carlevaro (1965, p. 29), que la Universidad en los últimos años había dedicado una creciente atención a la investigación científica: mayor asignación de recursos; extensión del régimen de dedicación total a todos los servicios universitarios; aumento en el número de docentes de tiempo completo y la creación de la Comisión Central de Investigación Científica que propició normas generales para el desarrollo de la investigación y distribuyó partidas para la ejecución de proyectos concretos. Pero era urgente "establecer los lineamientos de una política de desarrollo de la investigación científica en la Universidad y en el país"(p. 31). Para ello era ineludible saber con exactitud cuáles eran los recursos materiales y financieros

20 Carlos Quijano (1900-1984), era abogado, político, periodista, fue docente de Economía en la Facultad de Derecho, diputado por el Partido Nacional y fundador del semanario Marcha (1939).

21 Hermógenes Álvarez (1905-1984), era doctor en medicina, docente y director de la Clínica Ginecotológica de la Facultad de Medicina (1954-1970), consejero (1958-1961) y decano de la Facultad de Medicina (1966-1969). 
disponibles, ${ }^{22}$ a fin de, como había reclamado la Comisión, poder establecer las posibilidades del país en la materia y las lagunas, carencias o necesidades fundamentales a satisfacer.

En ese contexto el Plan de reestructuración de la Universidad (1967), cuyo fin era dar lugar a algunas de las viejas aspiraciones de los sectores reformistas de dicha institución, estaba pensado "en torno a la función de producción de conocimientos como tarea central de la Universidad" (Otero, 1969, p. 64-65). En él se sostenía que

[...] la investigación científica y tecnológica, base directa de una adecuada producción e industrialización nacional, no ha sido hasta el momento, convenientemente contemplada, sea en los planes universitarios, sea en los planes nacionales. Sin embargo, debe comprenderse que la investigación científica pura no es sólo un objetivo en sí mismo, dentro de la estructura universitaria, sino que, como fundamento de la investigación aplicada, es la base del progreso tecnológico sin el cual las naciones no pueden adquirir la adecuada prosperidad económica que está en la base de su bienestar social (Maggiolo, 1986, p. 7).

Sobre todo para naciones pequeñas como la nuestra, no era posible concebir

[...] un porvenir adecuado dentro de la región, [sin] promover un alto grado de tecnificación de su potencial humano. Este deberá ser capaz de competir en el campo internacional, por el poder del ingenio adecuadamente cultivado en la Universidad, luchando contra la desventaja que representa tener escasos recursos materiales y un minúsculo mercado interno (Ibid, p. 10).

Si dichas naciones querían salir de la condición de país productor de materias primas con bajo valor agregado, sólo tenían como camino posible cultivar la ciencia. La ciencia como un poderoso instrumento transformador de la realidad que permitiría convertir a un país como el nuestro en un productor de materia con alto valor agregado (Guarga en Markarian, Secco, Balás, 2017, p. 16).

El complejo tecnológico que una sociedad necesita [agregaba el PRU] no se satisface con la preparación de técnicos universitarios capaces de asimilar y aplicar los conocimientos existentes. Es necesario proporcionar al menos a un grupo bien seleccionado de ellos la formación de post-grado, que los habilite en la tarea creativa que permita a la sociedad uruguaya encarar con autonomía y solvencia los problemas que en el futuro se le irán presentando cada vez con mayor agudeza.

Tampoco se resuelve el problema preparando sólo profesionales del tipo clásico; hoy la sociedad necesita también físicos, economistas, historiadores, químicos, psicólogos y pedagogos de alto nivel científico y nuestra Universidad no los prepara en el momento actual (Maggiolo, 1986, p.11).

Como primera meta, el plan proponía la ampliación de la Universidad a través de un sistema organizado con el propósito de incentivar la investigación científica, en los centros ya existentes y en otros nuevos que contemplaran disciplinas que aún no se cultivaban en el país, o que se cultivaban en forma deficitaria.

22 "Es preciso tener un inventario de los recursos de todo tipo que se destinan a la investigación: recursos humanos, locales, bibliotecas, laboratorios, equipos, instrumental, así como los montos en dinero disponibles. Necesitamos también, de un modo orgánico y dinámico a la vez, identificar los centros, los núcleos de investigación existentes — con toda la variada y compleja apariencia que los centros de investigación pueden adoptar según la disciplina de que se trate- así como las personas que están trabajando en investigación, el tipo de función que desempeñan, el porcentaje de su tiempo que dedican a la investigación y el que destinan a sus demás tareas; los proyectos de investigación emprendidos por cada núcleo, el estado de avance de los mismos y los requisitos necesarios para proseguirlos, las nuevas investigaciones proyectadas y los recursos adicionales que ellas demandarán" (Carlevaro, 1965, p. 32). 
Particularmente, la formación e investigación en las disciplinas básicas debía perder el carácter de subproducto con respecto al objetivo principal de formar profesionales, para lo cual el camino más adecuado era el de descentralizar, de las respectivas facultades, los institutos de disciplinas básicas, de carácter netamente interdisciplinario, formando institutos centrales; a través de estos se pretendía, además, romper con la estructura federal de las facultades profesionalistas, evitar duplicaciones y conformar una organización adecuada modificando la pesada forma de administración existente.

Los centros propuestos eran conjuntos de facultades o facultades por sí mismas. Por su parte, los institutos, que tenían un importante carácter interdisciplinario, eran áreas que se entendía conveniente que salieran de las facultades en las cuales se habían ido desarrollando en forma un tanto espontánea, en general como resultado de iniciativas individuales. La enseñanza e investigación que en ellos se realizaría comprendería una cantidad de carreras.

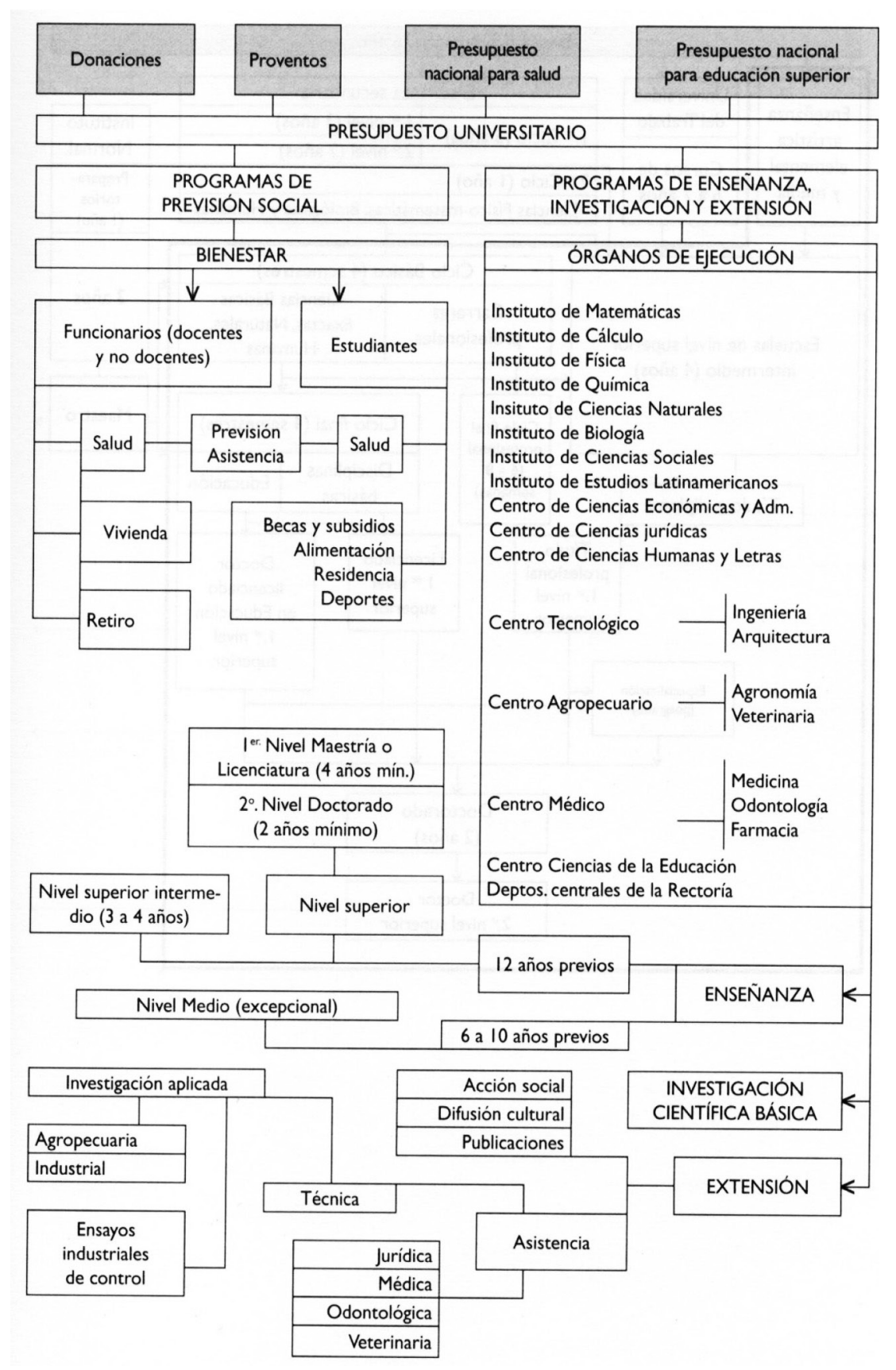

Diagrama I. (Maggiolo, 1986, p. 33) 
La creación de esos centros no representaba la desaparición de las facultades. Los primeros serían organismos que se reunirían unas pocas veces al año y cuyo fin consistiría en "planificar la formación de nuevos grupos de investigación, así como el fortalecimiento de los existentes”23 (Maggiolo, 1986, p. 12).

El estímulo de la investigación científica básica, que era señalado explícitamente como uno de los propósitos fundamentales del plan, se realizaría a través de las siguientes vías:

Formación de recursos humanos: la formación de investigadores al nivel de doctorado, ya fuera en el país o en el extranjero a través de la concesión de becas ${ }^{24}$ y eligiendo los temas a desarrollar de acuerdo a las necesidades del país y de la Universidad. Un objetivo fundamental del plan era formar recursos al nivel de doctorado en aquellas disciplinas que la Universidad considerara más necesarias. Como incentivo se les garantizaba trabajo en el propio instituto universitario, equipo y colaboradores una vez que su formación hubiere culminado. En aquellas disciplinas que no se enseñaban en la Universidad en ese momento, se comenzaría por formar jóvenes en el exterior, a nivel de master o licenciado, con el fin de acelerar el proceso de contar con un conjunto de personas preparadas en las disciplinas básicas.

Se contemplaba también fomentar el ingreso de profesores extranjeros (hasta 50) por períodos prolongados, para iniciar trabajos de investigación con grupos de jóvenes en aquellas disciplinas que no se practicaban aún o que existiera un interés particular en desarrollarlas.

De la misma forma, se posibilitaría los viajes de docentes (hasta 30) al exterior para asistir a congresos científicos y visitar centros importantes de investigación por períodos de hasta tres meses.

Por último, se proponía triplicar en el quinquenio el número de docentes con dedicación total (evitando así la emigración al sector privado, al Estado o el exterior) e incrementar el número de personal técnico y auxiliar de investigación.

Equipar con instrumental científico y material bibliográfico tanto los centros con nivel científico adecuado como aquellos a formar, a través de la financiación de programas concretos de investigación centralmente coordinados, que fueren presentados por los investigadores o por los respectivos servicios universitarios. Se consideraba de este modo, como auxiliares indispensables en esta transformación aspectos que hasta el momento habían sido desatendidos: servicios centralizados de bibliografía, instrumental y publicaciones.

Proyectar nuevos edificios universitarios contemplando las condiciones adecuadas para el desarrollo de tareas de investigación (Maggiolo, 1986, p. 16).

En cuanto a inversiones y equipos, el plan ya proponía una política de distribución por ramas que mostraba cierta orientación de qué campos interesaba estimular de acuerdo con la idea de desarrollo de país que se tenía. De un total de 7 millones de dólares a invertir en el quinquenio, 2 millones eran para las ciencias agropecuarias, 1,5 millón para ciencias técnicas, 1 millón para ciencias médicas, seiscientos mil para ciencias matemáticas, seiscientos mil para ciencias biológicas y naturales, seiscientos mil para ciencias físicas, cuatrocientos mil para ciencias químicas y trescientos mil para ciencias humanas.

Por otra parte, en línea con un artículo publicado doce años antes en la Revista de la AUPC, Maggiolo enumeraba en el $P R U$ los recursos necesarios para la investigación respetando el mismo orden: recursos humanos, equipamiento y edificios. Como en aquella ocasión también ahora manifestaba que, en los países latinoamericanos, se daba el proceso exactamente inverso: siempre era posible conseguir dinero para construir un gran edificio, luego conseguir un préstamo a muy bajo interés para equipamiento, "pero nadie parece acordarse [señalaba] de formar personas para que estén adentro de esos edificios y para que usen los equipos que se han conseguido..." (Universidad de la República, 1967, folio 897).

23 Los centros estarían constituidos por el decano y un consejero de cada orden de cada facultad. Su función principal era analizar todos los planes y programas de desarrollo y estudio que, debiéndose realizar en una de las facultades, tuviera repercusión en las otras.

24 Se planteaba promover el envío de 100 jóvenes becados al exterior para formarse en centros de investigación científica, seleccionados de entre 500 que usufructuarían becas internas destinadas a estudiantes universitarios que trabajarían en los centros de investigación existentes y en los que se fueran creando (Maggiolo, 1986, p. 26). 
A pesar de poner un énfasis especial en la formación de recursos humanos, Maggiolo advertía igualmente que no debían descuidarse los otros dos aspectos, porque ello podía representar trabajar para la exportación de investigadores.

Si formamos científicos y no les damos equipos, no les damos una remuneración adecuada y no les damos espacio para trabajar, lo que va a ocurrir es que el mercado internacional que existe para este tipo de personas los absorbe (Universidad de la República, 1967, folio 897).

En cuanto a la investigación científica aplicada, que el plan dividía en industrial y agropecuaria, no se diferenciaba sustancialmente de la investigación científica básica y correspondía financiarla con los recursos universitarios propiamente dichos o con proventos, según fuera el caso. Dicha investigación era considerada, además, un aspecto extensionista entre las funciones de la Universidad, que podía ser agrupada con la asistencia técnica que aquella brindaba. Los institutos o departamentos de las disciplinas aplicadas, en cada centro o facultad, debían inspirarse, según Maggiolo, en el modelo norteamericano más que en el modelo alemán. Este último era fundamentalmente un director que planificaba y desarrollaba todo el trabajo del instituto, y que dirigía a un conjunto de personas, normalmente de grado inferior. El modelo norteamericano, por su parte, tenía un sistema colegiado según el cual los docentes de mayor categoría ejercían colectivamente la dirección de la orientación en investigación y uno era elegido como administrador o coordinador, aunque no con el carácter de director de las tareas de investigación. Uruguay, aunque con cierto retraso, siguió los modelos constituidos por los centros científico-tecnológicos internacionales que se sucedieron en el liderazgo de esa actividad a lo largo de los últimos siglos. Inglaterra detentó la supremacía hasta la segunda mitad del siglo XVIII, siendo sucedida por Francia. Al comenzar el siglo XIX la ciencia francesa ocupaba un lugar de privilegio que fue perdiendo conforme avanzaba el siglo, fundamentalmente en relación con Alemania, debido a la capacidad del sistema alemán para modificarse de acuerdo con las necesidades y potencialidades de la investigación científica. ${ }^{25}$ Finalmente, ya en el siglo xx Alemania fue sustituida en el liderazgo por Estados Unidos. ${ }^{26}$

Los recursos previstos en el Plan se planteaban como los necesarios para una economía en desarrollo que requería, por lo tanto, una estructura educativa y de investigación en evolución. El documento daba las razones por las cuales la investigación debía estar en manos de la Universidad en una primera etapa, pero luego, parte de ella, sería descentralizada si se lograba previamente una infraestructura material y los recursos humanos necesarios para hacerlo así. Era un planteo a mediano y largo plazo, en tanto estrategia para la investigación científico-tecnológica y sus relaciones con el desarrollo de la economía.

El Plan, en consonancia con lo señalado, respecto al contexto nacional en el que venía a insertarse, hacía énfasis en la relación investigación científica-desarrollo, en la independencia económica y nacional a través del fomento de la ciencia y la tecnología y de la educación superior y en la integración regional. Características claras de un ideal desarrollista que por ese entonces marcaba a la región y que en Uruguay, según Garcé, se regía por una "simple y casi obvia filosofía: el crecimiento futuro alrededor de una expansión muy agresiva del país hacia el exterior” (2011, p. 36). El desarrollo económico y social requería remover trabas estructurales.

25 Los desarrollos económicos y políticos que pusieron al país germano en vías de industrialización hicieron que la ciencia y la tecnología fueran consideradas pertinentes para la resolución de problemas, y que las oportunidades para la innovación condujeran a la aparición de la instrucción regular y la formación de carreras de investigación científica. En ese contexto fue creada la primera escuela significativa desde el punto de vista de la institucionalización de la ciencia, la de Justus Liebig. Su gran innovación fue combinar enseñanza, investigación y producción en la misma institución. Así comenzó a surgir el papel del investigador profesional y la estructura de lo que llamamos modelo de laboratorio alemán, que combinaba las tres actividades mencionadas y nucleaba a grupos de trabajo alrededor de algún maestro de personalidad relevante, que seleccionaba una comunidad de fuerte vinculación y generaba una actividad sistémica.

26 La idea de que la investigación y la enseñanza en la universidad no podía determinarse más que por el estado de la ciencia y la creatividad de los docentes se puso en vigor más radicalmente en EEuU que en Alemania. El papel del científico como profesional y la estructura flexible de la universidad con su apertura a las innovaciones, introdujeron múltiples cambios en las relaciones que prevalecían hasta entonces entre la organización académica y las ciencias. A su vez, la transformación del nexo entre educación superior e investigación por una parte y la economía por otra, creó una demanda amplia y sin precedentes para los conocimientos y la investigación, lo que condujo a que la ciencia llegara a ser un recurso económico sumamente importante. 
Para ello era necesario: reforma agraria, promoción industrial, fortalecimiento de la infraestructura, reforma administrativa, reforma tributaria y planeamiento educativo, entre otros aspectos.

Respecto a este último punto es necesario señalar que durante las décadas de 1950-60 en América Latina cobró centralidad la naturaleza de la relación entre educación y desarrollo (D’Avenia, 2014, p. 148). La educación mereció un lugar privilegiado en la estrategia general de desarrollo económico y social. En nuestro país surgieron propuestas que entendieron a la educación como un elemento primordial en el proceso de desarrollo y señalaron un conjunto de fines prácticos característicos de cada uno de sus tramos visualizados desde un punto de vista expresamente pragmático, aunque sin desconocer la trasmisión cultural como objetivo propio de la educación.

El proceso de desarrollo crea nuevas funciones, necesita de la asimilación de la población al proceso, de mano de obra calificada a todos los niveles, de nuevas invenciones que provocarán el progreso técnico - elemento fundamental en el proceso-, de hábitos de consumo y de ahorro, de espíritu de empresa y de trabajo adecuados y así sucesivamente. Todos estos elementos se los demanda específicamente a la educación, que de esta manera deberá estar al servicio de las metas del desarrollo (Astori, Couriel, Santías y Zerbino, 1964, pp. 31-32).

Como consecuencia, los autores concluían la necesidad de darle un tratamiento prioritario a la inversión en educación en la asignación presupuestal yla importancia de una planificación que adecue educación y desarrollo.

El Plan de reestructuración de la Universidad inserto en este contexto, es un ejemplo de planificación en el que se solicita un determinado presupuesto y se establece, en contrapartida, la pretensión de cumplir ciertas metas u objetivos. A pesar de esto, el rector Maggiolo estaba en desacuerdo, en muchos aspectos, con algunos de los documentos políticos y económicos más importantes y representativos de este periodo-por ejemplo, el Plan Nacional de Desarrollo Económico y Social 1965-1974, formulado por la CIDE-. En el campo de la investigación científico-tecnológica que es lo que aquí nos atañe, discrepaba fuertemente con el sesgo profesionalista que proponía el plan, en desmedro de las funciones de investigación básica y aplicada. Como ya se ha señalado, de acuerdo con el espíritu del $P R U$, la Universidad estaba llamada a ocupar un lugar central en el desarrollo autónomo del país a partir del papel clave que jugaba en ese proceso la investigación científica y tecnológica, es decir, la creación de conocimiento original.

\section{Conclusión}

La Universidad de la República fue sensible a los distintos proyectos de país a los cuales acompañó, adaptándose a las diferentes necesidades que se imponían. Es así que la Universidad de fines del siglo Xix y principios del xx estuvo orientada fundamentalmente a la formación de los técnicos nacionales necesarios para apoyar el modelo de país moderno que se impulsaba, autónomo científica-tecnológica y energéticamente del exterior, con una industria nacional desarrollada e integrado al mercado mundial. Cuando este proyecto de país fue modificado y el interés se centró en otros objetivos, la economía comenzó a mirar al mercado externo y la idea de levantar una industria en el país fue dejada de lado y sustituida por la del Uruguay como vendedor de materia prima. La economía ya no buscó ampliar el mercado interno, sino el exterior, principalmente colocando allí carne y lana. Para vender esos productos en el mercado mundial era necesario cumplir con determinadas normas de calidad; el sistema educativo debía, por tanto, formar profesionales idóneos capaces de controlar que esas exigencias se cumplieran. Ese fue el papel de la universidad profesionalista, formadora de los cuadros profesionales de dirección.

Este trabajo se sitúa en el momento de crisis de ese modelo universitario en nuestro país y en el que se hacía fundamental énfasis, por parte de algunos sectores reformistas de la institución, en la necesidad de que la Universidad, además de formar profesionales, desarrollara tanto investigación científica básica como aplicada, con el objetivo de contribuir al desarrollo nacional. 
La Ley Orgánica de 1958 había democratizado el funcionamiento de la Universidad mediante el cogobierno de los tres órdenes, consagrando su autonomía administrativa, académica y presupuestal del poder político e incrementando el rango de acciones sociales de la institución. No generó, sin embargo, los cambios estructurales que permitieran mejorar su calidad académica. Permanecían sin resolución los debates sobre la reformulación de su estructura, particularmente en lo referente al lugar subalterno que ocupaba la investigación científica en una universidad que seguía estando conformada por una federación de facultades de marcada orientación profesionalista.

Es en ese contexto que Óscar Maggiolo accede al rectorado con la firme proposición de que durante su gestión se produjera un gran avance de la ciencia y la tecnología en dicha casa de estudios, y que desde allí se proyectara a todo el país revitalizando sus fuentes productivas.

El Plan de 1967, propio de una época caracterizada por el afán planificador, traducía en un presupuesto la misión de la Universidad: promover el desarrollo económico y social a partir del fomento de las investigaciones y carreras científicas y tecnológicas más vinculadas a la problemática económica del país. No en vano las ciencias básicas que se pretendía estimular eran aquellas que se consideraban imprescindibles para fundamentar el desarrollo nacional. La investigación científica es uno de los aspectos centrales del plan, no solo por su interés directo, sino porque es fundamento de la investigación aplicada, que a su vez es la base del progreso tecnológico sin el cual las naciones no pueden adquirir la adecuada prosperidad económica que está en la base de su bienestar social. La generación reformista de las décadas de 1950-60 defendía el rol de las instituciones de educación superior y la instauración de la institucionalidad científica como elementos fundamentales para el cambio social y visualizaba a la investigación científico-tecnológica como la clave para el logro de ese cometido.

Para el rector Maggiolo, particularmente, el futuro del país dependía de su desarrollo científicotecnológico. La autonomía cultural era condición necesaria para una verdadera independencia económica y política. Maggiolo vislumbraba un futuro auspicioso para la nación: "es fácil concebir que nos espera un porvenir adecuado dentro de la región, si podemos promover un alto grado de tecnificación en nuestro potencial humano" (Maggiolo, 1986, p.10). Para ello no había que confiar en seguir el curso de las naciones avanzadas, sino hacer camino propio. Maggiolo tenía claro que las soluciones a determinados problemas en ciertos contextos no sirven para resolver problemas en contextos diferentes.

El agente de este proceso, el responsable de promover la educación superior y el desarrollo de la ciencia y la tecnología era el Estado. Pero el futuro no dependía sólo de lo anterior. Era imprescindible, también, que el país no estuviera aislado, era necesario pensarlo en el contexto de la región. Tomar conciencia de que Uruguay no podía pensar su futuro sin preocuparse por su porvenir científico; y tomar decisiones acordes con la gravedad de la situación definiría el destino del país dentro del concierto mundial y continental.

En un sentido estricto el Plan Maggiolo fracasó. Su inviabilidad se debió tanto a razones internas como externas a la institución. Sin embargo, el documento en sí mismo tiene un valor innegable. Constituyó el desafío de pensar por primera vez a la Universidad como una institución global y de proponer una reestructuración integral, en relación con las necesidades del país. Pretendió no solamente dar respuesta a interrogantes como: ¿para qué debería servir la Universidad?, al menos nuestra Universidad, ¿cuál es su deber con la sociedad?, ¿cuál es o debería ser el rol transformador de la educación superior?, sino que, además, propuso una serie de medidas y mecanismos, es decir, elaboró un plan y asignó recursos, a través de los cuales esas respuestas pudieran concretarse en la práctica. Propuso una estrategia para que la Universidad cumpliera con su misión más cara: contribuir al desarrollo y la transformación social, a la autonomía científico-tecnológica y cultural como fundamento de una independencia real del país.

El plan daba cuenta así, aunque de una manera original, de una preocupación extensamente difundida en ese momento en América Latina: la relación ciencia, tecnología y desarrollo. Una universidad que aportara al desarrollo nacional mediante la formación en ciencia y tecnología estaba en el foco de las corrientes desarrollistas de la época. 
Es cierto que la propuesta de Maggiolo puede ser calificada de optimista: confiaba en que era posible transformar la estructura universitaria y cumplir con tareas que no se estaban cumpliendo o no se lo hacía adecuadamente. Fue optimista en demasía, seguramente, al asumir que bastaba con mostrarle al industrial lo que la ciencia y la tecnología nacionales podían hacer por su industria para que tomara conciencia e invirtiera en el desarrollo de esa actividad en el país.

Sin embargo, cincuenta años después el Plan Maggiolo sigue inspirando, pero sobre todo interpelando a la Universidad uruguaya. Aunque la institución ya no forma solamente profesionales, sino que forja investigadores en las distintas ciencias básicas y aplicadas, a pesar de que se han creado nuevas facultades y centros de investigación y aunque actualmente ya no es solamente un grupo de reformadores el que lucha por promover el desarrollo científico y tecnológico del país como clave para su futuro, aún quedan por cumplir muchos de los desafíos que Maggiolo avizoraba.

\section{Bibliografía}

ASTORI, Danilo; Couriel, Alberto; Zerbino, Ricardo y Santías, José.Vinculación entre educación y desarrollo económico y social, Montevideo: Facultad de Ciencias Económicas. Tesis de grado, 1964.

CARLEVARO, Domingo. Recursos para la investigación científica en la Universidad de la República.Galileo. Montevideo, n. ${ }^{\circ}$ 2-3, p. 29-33, 1965.

CASSINONI, M. Memoria del Rectorado 1957-1960. Montevideo, 1962.

CHERONI, Alción. Políticas científico-tecnológicas en el Uruguay del siglo xx. Temas de Nuestro Tiempo/9. Montevideo:Facultad de Humanidades y Ciencias. Universidad de la República, 1988.

CHERONI, Alción. Políticas en ciencia, tecnología e innovación en el Uruguay de los siglos Xx y xxi. Un análisis epistemológico desde la perspectiva del materialismo dialéctico. Serie Ponencias del Taller PT 02/10. Grupo Interdisciplinario "Estado, Sociedad y Economía" en los siglos XX Y XXI (GIESE 2021) de la Universidad de la República. Montevideo, 2010.

Comisión Especial para Fomento de Laboratorios Tecnológicos e Investigaciones Industriales. Antecedentes relacionados con la creación en el Uruguay de un Centro de Asistencia Técnica para la Industria. Montevideo, 1956.

D’AVENIA, Lucas. Desarrollismo y educación en Uruguay en los 60. Aproximación a la producción de conocimiento sobre educación y a la agenda de la política educativa de la CIDE, Contemporánea. Historia y problemas del siglo XX. Montevideo, 5, n. ${ }^{\circ 5}$, 2014. p. 147-166.

GARCÉ, Adolfo.Ideas y competencia política en Uruguay (1960-1972). Revisando el "fracaso" de la CIDE. Montevideo: Trilce, 2002.

GARCÉ, Adolfo. Investigación y políticas públicas. Planes de desarrollo en Uruguay en tiempos de la Alianza para el Progreso, Contemporánea. Historia y problemas del siglo Xx. Montevideo, 2, n. ${ }^{2} 2,2011$, p. 31-50.

GARCÍA, V., Maggiolo. O y Ricaldoni, J. Consideraciones sobre la creación del Centro de Investigaciones Tecnológicas. Archivo Laguardia (Caja 1. Carpeta1.6.) Montevideo: Archivo General de la Universidad, 1956.

GROMPONE, Juan. Para una historia de las ciencias en el Uruguay: seis artículos. Galileo, Montevideo,n. ${ }^{0}$ 16, 2a Época, 1997, p. 27- 53.

GROMPONE, Juan. Informe presentado al Foro sobre Política de la Investigación Científica de la Universidad de la República, Distribuido N. ${ }^{\circ}$ 319/970. Oficina de Publicaciones del Centro de Estudiantes de Humanidades y Ciencias (CEHC), Montevideo, 1970, p. 1-16.

LATAPÍ, Pablo.Algunas tendencias de las universidades latinoamericanas. México: Unesco-AIU, 1976.

MAGGIOLO, O., Posada I., Martínez, J. y Bouton, A. El problema de la Facultad de Ingeniería. Montevideo: Peña, 1944.

MAGGIOLO, Óscar. La ciencia, la técnica y la sociedad actual.Boletín de la Asociación Uruguaya para el Progreso de la Ciencia. Montevideo, vol. 1, n. ${ }^{\circ}$ 1, 1952, p. 5- 11. 
MAGGIOLO, Óscar. La enseñanza de la ciencia en el ciclo secundario. Boletín de la Asociación Uruguaya para el Progreso de la Ciencia. Montevideo, vol. 2, n. ${ }^{\circ}$ 1-2, 1953, p. $1-2$.

MAGGIOLO, Óscar. Recursos y hombres para la investigación científica.Revista de la Asociación Uruguaya para el Progreso de la Ciencia. Montevideo, vol. 4, n. ${ }^{\circ}$ 1, 1955, p. 1-3.

MAGGIOLO, Óscar. La ley orgánica de la Universidad. Revista de la Asociación Uruguaya para el Progreso de la Ciencia. Montevideo, vol. 5, n. ${ }^{\circ} 1,1958$, p. 1-3.

MAGGIOLO, Óscar. Política de desarrollo científico y tecnológico de América Latina. Temas de nuestro tiempo/4. Montevideo: Facultad de Humanidades y Ciencias. Universidad de la República, 1985.

MAGgiolo, Óscar. Plan de reestructuración de la Universidad. Montevideo: Universidad de la República, División Publicaciones y Ediciones, 1986.

MARKARIAN, Vania; Jung, María Eugenia; Wschebor, Isabel.1968. La insurgencia estudiantil. Aniversarios 2008, vol. 4. Montevideo: Universidad de la República. Archivo General, 2008.

MARKARIAN, Vania; Secco, Lucía; Balás, Mariel. Lo que Maggiolo proponía no era una quimera. Entrevista a Rafael Guarga. InterCambios.Montevideo, vol. 4, n. ${ }^{\circ}$ 1, 2017, p. 13-18.

MARTíNEZ, María Laura.La Asociación Uruguaya para el progreso de la ciencia.Galileo. Montevideo, n. ${ }^{\circ} 23,2 .^{\text {da }}$ época, 2001, p. 17-34.

MARTÍNEZ, María Laura. El proyecto Eduardo Acevedo. La política científica y tecnológica en el primer batllismo. En Fondo Bicentenario "José Pedro Barrán". Políticas científicas, tecnológicas y de innovación en el Uruguay contemporáneo (1911-2011). Montevideo:ANII, 2011, p.15-87.

MARTíNEZ, María Laura.75 primeros años en la formación de los ingenieros nacionales. Historia de la Facultad de Ingeniería (1885-1960).Montevideo: Facultad de Ingeniería, Universidad de la República, 2014.

Memoria- Armour Research Foundation-Plan Armour de Ayuda Técnica Internacional al Uruguay. Revista de la Unión Industrial del Uruguay. Montevideo, vol. 52, n. ${ }^{\circ 59,}$ 1950, p. 673-676.

OTERO, Mario. El sistema educativo y la situación nacional. Nuestra tierra. Montevideo, n. ${ }^{7}$ 7, 1969.

PARIS, Blanca.La Universidad de la República. Desde la crisis a la intervención. 1958-1973. Montevideo: Ediciones Universitarias, 2010.

Universidad de la República (Uruguay). Libro de actas del Consejo Directivo Central, Acta N. ${ }^{\circ}$ 27, folios 882-912, 1967.

Universidad de la República. Facultad de Ingeniería (Uruguay). Óscar Maggiolo. Reflexiones sobre la investigación científica. Montevideo: Mastergraf, 2009.

Universidad de la República. Archivo General (Uruguay). Universidad, investigación y compromiso. Documentos del Archivo Maggiolo. Montevideo: Universidad de la República. Serie "Archivos Privados en el AGU”, n. 4, 2010. 